\title{
NTPDase5/PCPH as a New Target in Highly Aggressive Tumors: A Systematic Review
}

\author{
Paula Andreghetto Bracco, Ana Paula Santin Bertoni, and Márcia Rosângela Wink \\ Laboratório de Biologia Celular, Departamento de Ciências Básicas da Saúde, Universidade Federal de Ciências da Saúde de \\ Porto Alegre (UFCSPA), Rua Sarmento Leite, Rua Sarmento Leite, No. 245, 90050-170 Porto Alegre, RS, Brazil
}

Correspondence should be addressed to Márcia Rosângela Wink; marciawink@yahoo.com.br

Received 26 February 2014; Accepted 18 May 2014; Published 23 June 2014

Academic Editor: Zhen Chen

Copyright (C) 2014 Paula Andreghetto Bracco et al. This is an open access article distributed under the Creative Commons Attribution License, which permits unrestricted use, distribution, and reproduction in any medium, provided the original work is properly cited.

\begin{abstract}
The protooncogene $P C P H$ was recently identified as being the ectonucleoside triphosphate diphosphohydrolase 5 (ENTPD5). This protooncogene is converted into an oncogene by a single base pair deletion, resulting in frame change and producing a premature stop codon, leading to a mutated protein (mt-PCPH) with only $27 \mathrm{kDa}$, which is much smaller than the original $47 \mathrm{kDa}$ protein. Overexpression of the PCPH as well as the mutated PCPH increases the cellular resistance to stress and apoptosis. This is intriguing considering that the active form, that is, the oncogene, is the mutated PCPH. Several studies analyzed the expression of NTPDase $5 / \mathrm{mt}-\mathrm{PCPH}$ in a wide range of tumor cells and evaluated its role and mechanisms in cancer and other pathogenic processes. The main point of this review is to integrate the findings and proposed theories about the role played by NTPDase $5 / \mathrm{mt}-$ $\mathrm{PCPH}$ in cancer progression, considering that these proteins have been suggested as potential early diagnostic tools and therapy targets.
\end{abstract}

\section{Introduction}

The ectonucleoside triphosphate diphosphohydrolase 5 (NTPDase5; EC: 3.6.1.6), also known as CD39L4 (CD39 antigen-like 4) [1], is an enzyme that acts mostly on diphosphate nucleosides, rather than triphosphate nucleosides. It can be secreted from mammalian cells through an aminoterminus hydrophobic domain that encodes a signal peptide sequence [2]. The CD39L4 has three potential glycosylation sites and has the capacity to form disulfide dimers; however, none of these characteristics seem to significantly contribute to the ADPase or UDPase activity, which distinguishes the CD39L4 from the other proteins in this family [3]. The NTPDase5 also differs from the other members of E-NTPDase family, as it is the only one characterized as a protooncogene, also known as PCPH $[4,5]$.

The $P C P H$ is a highly conserved gene from yeast to humans, suggesting basic roles in eukaryotic cells function [6]. It is located on chromosomes 12 and 14 in mice and humans, respectively, and it is transformed into an oncogene, called $m t-P C P H$, through a single base pair depletion resulting in frame change and producing a premature stop codon, originating a protein with 246 amino acids $(25 \mathrm{kDa})$ instead of the normal protein with 469 amino acids $(47 \mathrm{kDa})$ [7].

In the first studies, it was not possible to conclude whether the PCPH had nucleotide degrading activity, since it shares the apyrase conserved regions [7]. In 2001, Páez and collaborators showed a degree of genetic similarity of 98\% between the protooncogene PCPH and ENTPD5 and also determined that PCPH and NTPDase5 were capable of degrading ADP and ATP (preferentially ADP) [4]. First, it was shown that extracts from stable mt-PCPH-transformed NIH3T3 cells presented GDPase activity, using in-gel activity (performed by native gel electrophoresis of the extracts and staining for inorganic phosphate) and standard GDPase assays [8]. Later, the same group, by a different experimental approach, demonstrated that NTPDase activity of mt-PCPH was undetectable in vitro or when tested in situ in living cells [9]. The authors stated that this discrepancy could be due to the production of recombinant $\mathrm{mt}-\mathrm{PCPH}$ in non-human expression systems. 
The $P C P H$ gene was initially identified as a protooncogene, due to its frequent mutation in the 3-methylcholantrene-induced tumorigenesis process in a Syrian hamster fibroblast cell line [6]. Subsequent studies were mainly based on the hypothesis that the carcinogenic action of PCPH/NTPDase5 could modulate the cellular balance of ATP, potentially interfering with cellular functions such as cell cycle, apoptosis, autophagy, proliferation, and differentiation [5].

The major functional difference between the normal NTPDase 5 and the mt-PCPH oncoprotein is that the former provides lower levels of protection against apoptotic agents, including chemotherapeutic drugs, and radiation than the latter. This role of mt-PCPH seems to be mediated by its ability to promote a Ras-independent sustained activation of the ERK pathway $[7,8,10]$. The different expression profiles of these proteins and consequent changes in the cellular resistance observed in the studies reviewed suggest that it may be involved in the survival, growth, or migratory activity of some kinds of tumors. Yet, it is not very clear how this protein, in fact, contributes to the development of the different types of neoplasias. In addition, it was observed that NTPDase $5 \mathrm{KO}$ mice presented an increase in cases of liver pathology and neoplasia [11], adding more doubts about how the level of its expression influences cancer development.

The aim of this work is to provide an overview, in a systematic review format, of the expression profile of the NTPDase5 and mt-PCPH in cancer cell lines and tumor samples, in comparison to healthy tissues, and describe the proposed mechanisms by which the mutated and WT proteins act in the neoplastic development.

\section{Method}

This review describes a "literature overview" about PCPH/ NTPDase 5 in all types of cancer.

2.1. Search Details. We performed an electronic search on January, 2014, for papers indexed in PubMed and Scopus database. The search strategy comprised only the medical subject heading (MeSH) term "ENTPD5 or NTPDase5." For inclusion in this review, papers had to describe any relation of PCPH/NTPDase5 to cancer. No language restriction was applied. By this search strategy, 55 papers were identified. After reviewing their abstracts, 14 eligible papers were chosen and three citations retrieved from manual search were included, providing 17 papers that examined PCPH/NTPDase5 expression status in cancer (Figure 1).

Data were extracted from each original study about PCPH/NTPDase5 gene or protein expression in normal and pathological state as well as its isoform expression patterns in tissues and tumor cells.

\section{Results and Discussion}

3.1. Expression Profile of ENTPDase5. ENTPD5 is expressed at different levels in several types of normal and neoplastic tissues suggesting a tissue-specific regulation [12, 13]. Interestingly, the first studies on this subject revealed the presence of $27 \mathrm{kDa} \mathrm{PCPH}$ immune-related polypeptide (believed to be the truncated, i.e., mutated, $P C P H$ oncogene) in various cell extracts of healthy epithelial tissues and the expression of $47 \mathrm{kDa}$ polypeptide (believed to be the full length $P C P H$ gene), in most of the tumor cell lines studied, hypothesizing that there was a potential relationship between the levels of these proteins and the neoplastic phenotype [12]. However, high levels of the normal NTPDase 5 have also been identified in healthy liver and kidney tissues [13]. It is important to mention that these studies were performed with an antiPCPH polyclonal antiserum raised in rabbits with a purified bacterial recombinant $\mathrm{PCPH}[8,12,13]$. Considering that the PCPH/NTPDase5 shares the conserved apyrase regions [1], it is possible that this antiserum also recognizes other members of the E-NTPDase family.

Thus, it is possible to conclude that the expression of ENTPD5/mt-PCPH in tumors studied so far is highly variable, as described in Tables 1 and 2.

Breast tumors induced in mice exhibited increased expression of the normal NTPDase 5 and decreased expression of the mt-PCPH when compared with healthy mammary gland, and this difference was more evident in malignant tumors in comparison to benign tumors [13]. A similar expression profile was observed when comparing neoplastic cell lines of the larynx and healthy cells from a primary culture of the larynx. A higher mt-PCPH expression was observed in the healthy cells when compared with carcinoma cells lines: UM-SSC-11, UM-SCC-12, UM-SCC-23, and SCC81B. Interestingly, a loss of this protein expression was associated with an increase in the expression of the normal NTPDase5 [19]. In human normal and neoplastic breast samples, however, the normal NTPDase5 appeared in all tested samples both benign and malignant. Expression of $\mathrm{mt}-$ PCPH correlated positively with the aggressiveness of the breast carcinoma and was not detected in the benign tumors [18].

Testicular germ tumor cell lines NCCIT and NT2/D1 also presented high levels of NTPDase5 and very low levels of mt-PCPH [17]. However, most clinical specimens of germ tumor cell of 54 patients showed an increase in expression of mt-PCPH when compared with healthy adjacent tissue [17]. Additionally, the expression of $\mathrm{mt}-\mathrm{PCPH}$ is increased in the precursor lesions of germ tumor cell.

Immunohistochemical analysis also identified that, in cases of laryngeal cancer and testicular germ tumor cells, the concentration of NTPDase 5 is higher in areas of differentiation and neoplastic transformation, with low proliferation, than in areas with high proliferation rates, raising the hypothesis that this protein acts more in the initial processes of cancer than in well-advanced, malignant neoplastic phenotypes $[17,19]$.

Studies with clinical specimens of prostate cancer and prostate tumor cell lines RWPE-1, LNCaP, C4-2, and PC-3 demonstrated that NTPDase5 is not significantly expressed in healthy prostate tissue but is present in cases of benign hyperplasia and is more pronouncedly expressed in tumor samples. Also, this protein was not detected in the tumor 


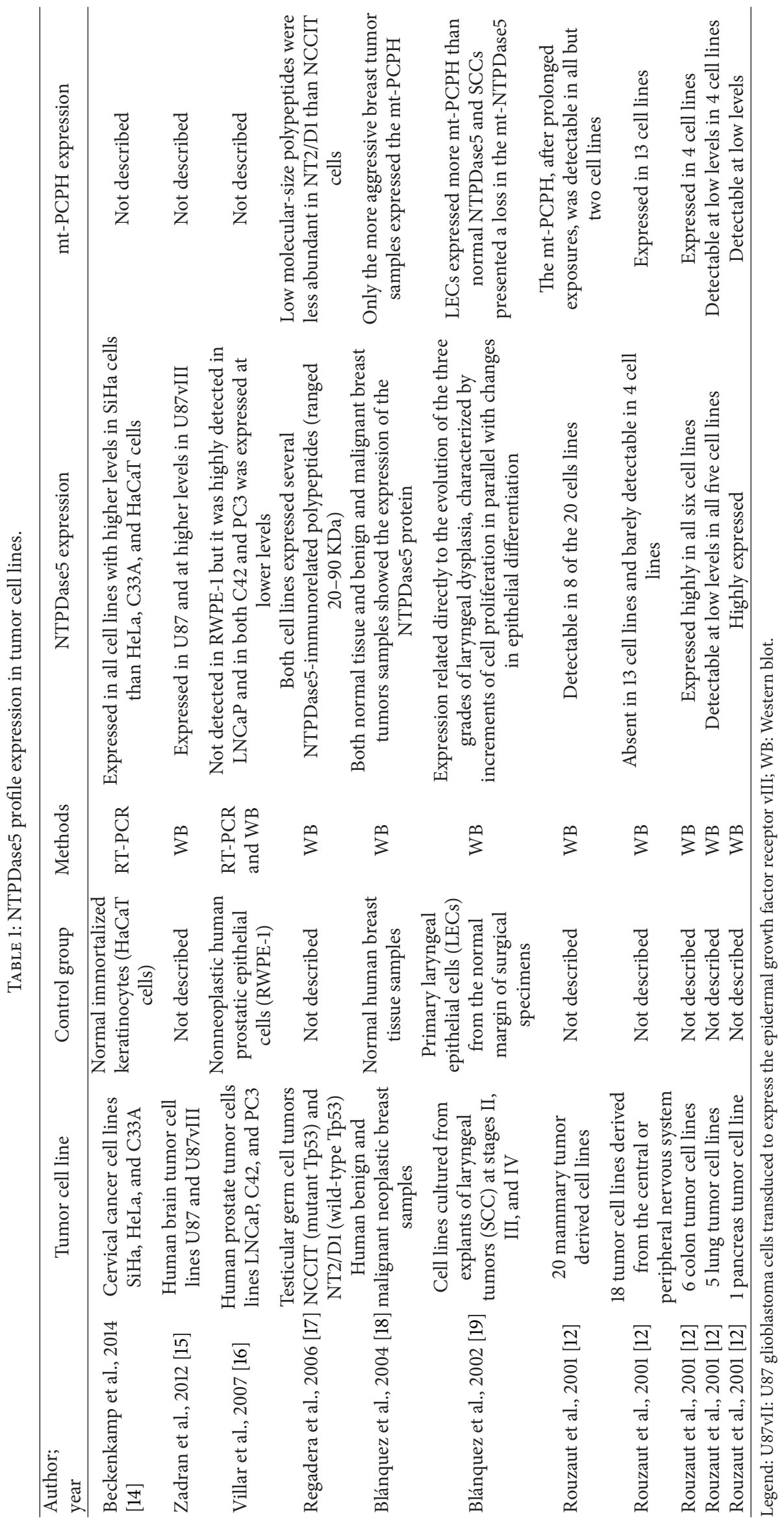




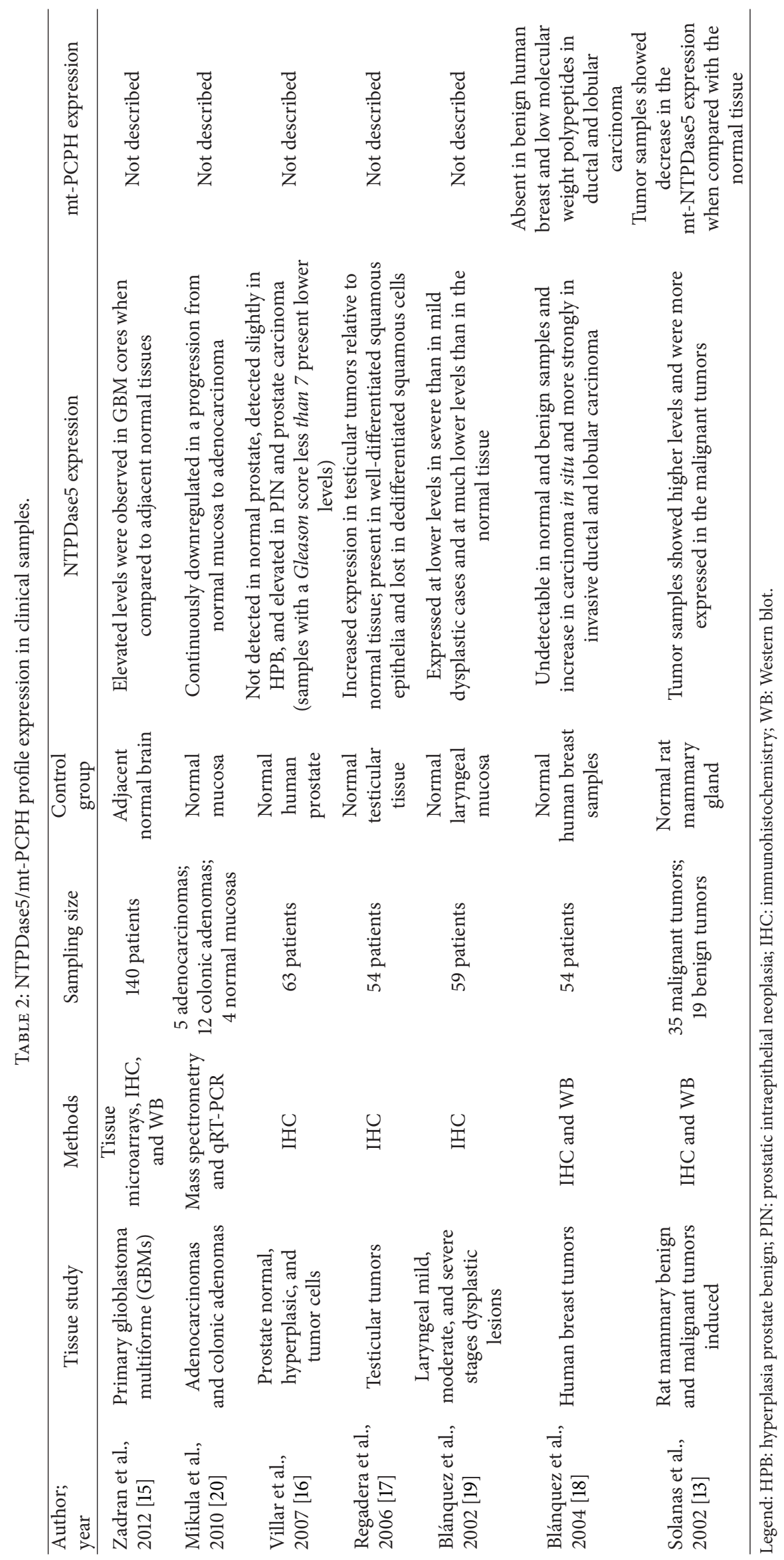




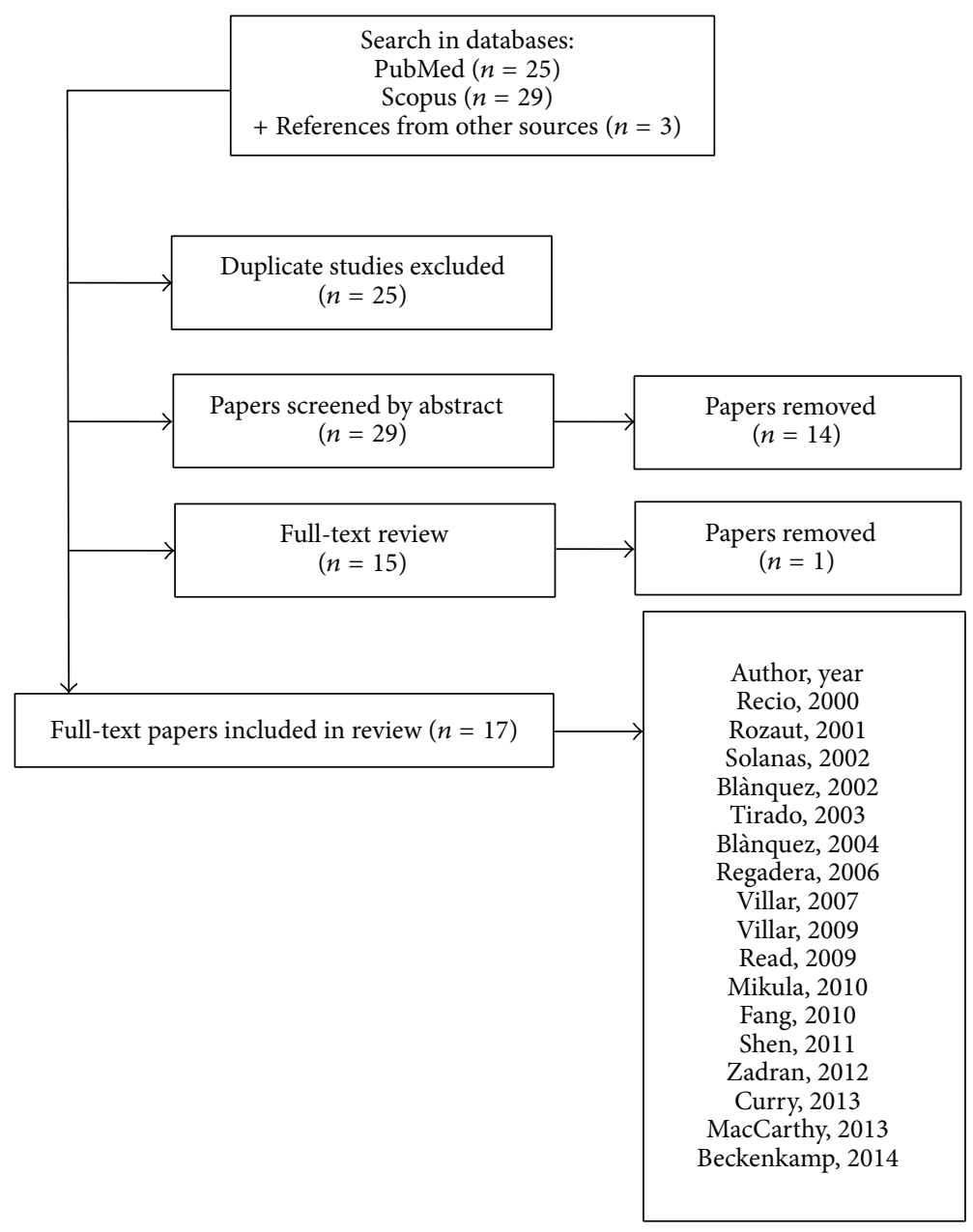

Figure 1: Methodological flow chart of the search strategy in PubMed and Scopus databases.

cell line PC-3 but was expressed in LNCaP cell line. In addition, a positive relationship between the level of NTPDase 5 expression, and especially mt-PCPH expression, and the invasiveness of prostate cancer was found, thus associating the expression of the NTPDase 5 more with cancer motility than with its proliferation [16].

Clinical samples of glioblastoma multiforme (GBMs) showed a higher level of NTPDase5 expression when compared to adjacent normal tissues [15]. In addition, the samples with higher expression levels of this protein were associated with significantly lower survival rates when compared to samples with lower NTPDase5 expression. However, the NTPDase 5 expression profile in individual samples of normal colon, adenomas, and colon adenocarcinomas presented that the ENTPD5 gene expression decreased with the increase of lesion malignancy [20].

When an increase in either the NTPDase 5 or the mt$\mathrm{PCPH}$ expression was present, this was observed as early as nonneoplastic lesions, suggesting that the deregulation of these proteins is involved in the initial stages of neoplastic development. Thus, it is possible to consider the use of the NTPDase5 as a tool for early identification of various neoplastic cells $[13,16,17]$.
Recently, cervical human cancer cells SiHa (HPV 16positive), HeLa (HPV 18-positive), and C33A (HPV-negative) were shown to present different levels of ENTPD5 gene expression, and the highest expression observed in SiHa cells suggests a link between ENTPD5 and oncogenic viral proteins in cervical cancer development [14].

3.2. How NTPDase5 Acts in Cancer Progression. The neoplastic transforming activity is the role of the mt-PCPH that represents the major functional difference between the normal protooncogene and mutated active oncogene. It is supposed that this activity is due to the ability of this protein to cause a Ras-independent sustained activation of ERK1 [8].

Although the normal NTPDase5 lacks the transforming ability, it is suggested that the NTPDase 5 and especially the mt-PCPH confer resistance to cells subjected to stress conditions [7]. Such resistance is conferred in part by the diphosphohydrolase activity of this enzyme, which causes a reduction in the intracellular ATP and inactivation of the stress-activated protein kinases, which is reversed by returning the intracellular ATP to physiological levels [21].

One of the cell protection mechanisms against apoptosis afforded by NTPDase 5 is through inhibition of mTOR. 
After cellular exposure to ionizing radiation, mTOR plays a proapoptotic role and this role is antagonized by the expression of $\mathrm{mt}-\mathrm{PCPH}$ protein or by the overexpression of the normal protein NTPDase5. They are responsible for blocking the activation of mTOR and its translocation from the cytoplasm to the nucleus, preventing the phosphorylation of p53 at $\operatorname{Ser}^{18}$. Phosphorylation of p53 mediates the release of cytochrome $\mathrm{c}$ by mitochondria and the subsequent activation of caspase 9/3, inducing the apoptosis [8]. This signaling pathway is influenced by intracellular ATP concentrations, and an increase in the expression of NTPDase 5 and mt$\mathrm{PCPH}$ blocks this pathway and reduces the levels of apoptosis (Figure 2).

It was also observed that the overexpression of the NTPDase5 protein and more significantly the mt-PCPH actually decreases the intracellular concentration of ATP and confers resistance not only to stress-induced apoptosis but also to those induced by chemotherapy. The overexpression of these enzymes increased resistance of prostate tumor cells when in contact with cisplatin and of colorectal carcinoma cells when in contact with oxaliplatin $[9,22]$.

The proposed mechanism by which NTPDase5/mt$\mathrm{PCPH}$ increases the neoplastic cell resistance to cisplatin is due to the ability of this protein to prevent the dephosphorylation of the kinase PKC $\alpha$ induced by chemotherapy. By keeping the PKC $\alpha$ phosphorylated at Thr638, this protein phosphorylates and stabilizes the antiapoptotic protein Bcl-2 at $\mathrm{Ser}^{70}$, making this enzyme resistant to cisplatininduced proteasome degradation pathway, as suggested by Villar et al. [22]. Furthermore, it was observed that NTPDase 5 interacts functionally with not only the PKC $\alpha$ but also the $\mathrm{PKC} \delta$ protein, which was recognized as a key mediator in the NTPDase5 functions related to changes in the cell growth and invasive activity of the pancreatic tumor cells (Figure 2) [22].

Fang et al. [23] have demonstrated the involvement of the NTPDase 5 also as an important link in the PI3K/PTEN loopsignaling pathway, which promotes cell growth and survival and is frequently found active in tumor cells. In this work, the authors found that PTEN knockout cells had increased expression of NTPDase5, and its overexpression is correlated with the activation of AKT, especially in cell lines of various tumor types and in primary tumor samples (Figure 2). These knockout cells also showed an increased degradation of ATP to AMP; however, as the substrates of NTPDase 5 are UDP and ADP, it was found that the ATP degradation pathway occurs in the presence of the enzyme CMPK1, which removes a phosphate of ATP to phosphorylate an UMP molecule, generating UDP and ADP, respectively.

NTPDase5, by activating AKT, also plays a critical role in triggering the Warburg effect, leading to an increase in anaerobic glycolysis even in the presence of oxygen, increasing the levels of lactate and production of important macromolecules for cell proliferation, promoting angiogenesis and metastasis $[23,24]$. Cells with activated PI3K/AKT pathway have a higher level of protein translation, which causes an overloading in the endoplasmic reticulum and increases the chances of a deficient folding process and consequent malformed proteins. The NTPDase 5 is a protein present in the endoplasmic reticulum, and overexpression of this enzyme increases the degradation of UDP to UMP promoting protein $\mathrm{N}$-glycosylation and folding, reducing the stress in the tumor cells endoplasmic reticulum $[23,24]$. This mechanism allows growth factor receptors such as EGFR, IGFR, and HER-2 to be expressed and properly folded, maintaining high levels of these receptors in tumor cells. In fact, NTPDase 5 knockout in cells possessing active AKT impairs the expression and glycosylation of these receptors $[23,24]$.

In addition to participating in the PI3K/PTEN signaling pathway, which is overactive in approximately $90 \%$ of GBMs, NTPDase 5 also plays an important role in the development of this cancer. This protein plays a modulatory role in the bioenergetics of this malignancy, increasing the catabolic efficiency of the aerobic glycolysis. In addition, when NTPDase5 is suppressed, it causes a decrease in fatty acid oxidation and promotes an increase in the ATP influx and of the autophagic vacuoles in the cytoplasm [15].

Tumors of the respiratory system have a heterogeneity in what concerns the activation of Akt and PTEN inhibition. Those with this signaling pathway being active, however, are more resistant to treatments that involve starvation [25]. Indeed, suppression of the NTPDase 5 in lung carcinoma cells results in a decrease in Akt activity, decreased levels of IGF-IR growth factor receptor, and reduced cell proliferation under conditions of starvation, making these cells more susceptible to this type of treatment [25].

Finally, overexpression of $m t-P C P H$ in colorectal carcinoma caused an increase in chemotherapy resistance [9]. The authors showed that a crucial event for this observation was a decrease in the intracellular levels of ATP [9]. However, no ATPase activity was observed in the mt-PCPH protein, probably due to a loss of conserved catalytic determinant regions in the truncated form, which may affect its tertiary structure and then the enzymatic function. This suggests that ATP degradation caused by the mutated protein may occur through interaction with other proteins and not through its NTPDase activity per se. Such findings can redirect all research involving the action of $\mathrm{mt}-\mathrm{PCPH}$ in neoplastic processes and need to be further investigated [9].

\section{Conclusions}

In most of the types of cancers studied, the NTPDase $5 / \mathrm{mt}$ PCPH shows a change in its expression levels even in precursors of the malignant and benign lesions, which makes this protein a potential tool for early diagnosis of tumorigenesis. This enzyme has also been identified as a key element in a number of pathways known to be frequently activated in neoplastic processes and which give tumor cells a survival advantage when compared to healthy cells. In most cases, the participation of the NTPDase $5 / \mathrm{mt}-\mathrm{PCPH}$ occurs with a change in the intracellular ATP concentration and with participation of this enzyme in the phosphorylation and activation processes of proteins with antiapoptotic activity, conferring to the tumor cells resistance against apoptosis by stress or by chemotherapy treatments. The two main 


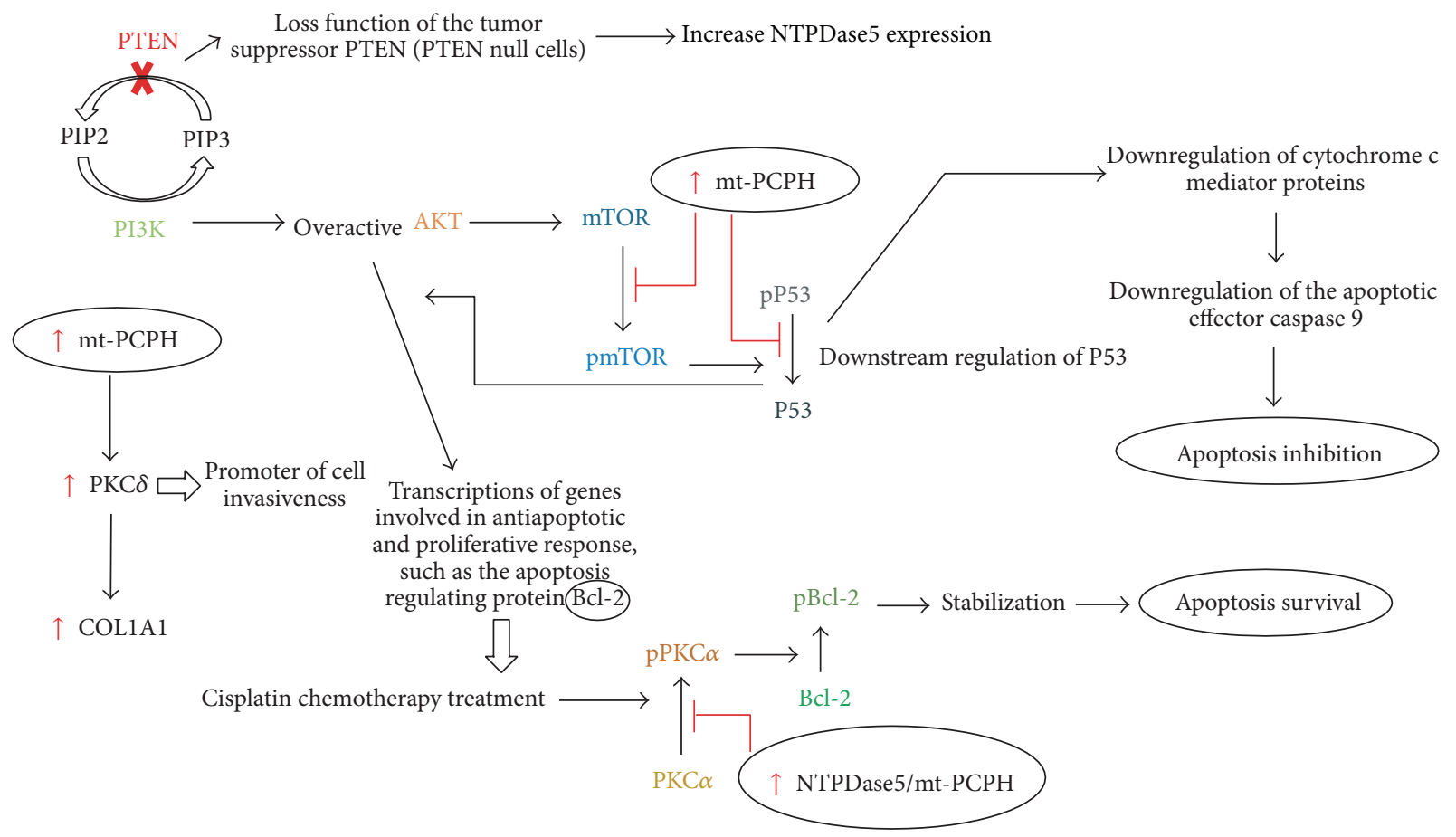

FIGURE 2: Integration of the proposed pathways by which the NTPDase5/mt-PCPH acts on the neoplastic progression. Due to the lack of information about the role of these proteins in cancer development and progression, this scheme presents all data published so far, not taking into consideration in which cell the proposed mechanisms were studied although it is possible that some of the contradictions presented may be a direct consequence of this fact. The figure demonstrates how the loss of the tumor suppressor PTEN possibly causes an increase in NTPDase5 expression and an overactive PI3K/AKT pathway. AKT and mTOR regulate cell growth and survival, such as Bcl-2 gene leading to an increase in apoptotic resistance. Furthermore it is also suggested that the NTPDase5 interacts with PKC $\delta$, upregulating its levels and inducing cancer cell invasiveness. pP53, pPKC $\alpha$, and $\mathrm{pBcl}-2$ are the phosphorylated forms of the respective proteins and correspond to the phosphorylation of $\mathrm{p} 53$ at $\mathrm{Ser}^{18}$, pPKC at $\operatorname{Trh}^{638}$, and Bcl-2 at Ser ${ }^{70}$.

pathways related to the NTPDase5/mt-PCPH activity are the mTOR and the PI3K/PTEN signaling pathways, which are directly related, since the inhibition of PTEN results in a PI3K and consequent $\mathrm{AKT}$ overactivation, which in turn regulates the growth of tumor cells by different signaling pathways, one being its effect on mTOR (Figure 2).

This review included all the data published so far regarding the role of the proteins NTPDase $5 / \mathrm{mt}-\mathrm{PCPH}$ in cancer development and progression. Due to the scarcity of studies with NTPDase 5/mt-PCPH, it is difficult to establish tissue- or cell-type-specific functions. However, it seems relatively well established that ENTPD5 and mainly $m t-P C P H$ expression are related with tumorigenic transformation. The findings presented in the studies reviewed raise the idea of using NTPDase 5 as a possible target for cancer treatment.

\section{Conflict of Interests}

The authors declare that there is no conflict of interests regarding the publication of this paper.

\section{Acknowledgments}

The authors thank Dr. Guido Lenz (Laboratório de Sinalização e Plasticidade Celular, UFRGS) for the critical revision of the paper and valuable corrections. Paula Andreghetto Bracco was a recipient of a Master fellowship from CAPES (Coordenação de Aperfeiçoamento de Pessoal de Nível Superior); Márcia Rosângela Wink is recipient of research fellowship from $\mathrm{CNPq}$ (Conselho Nacional de Desenvolvimento Científico e Tecnológico); Ana Paula Santin Bertoni is recipient of CAPES-PNPD fellowship. This study was supported by the Pronem-FAPERGS (Fundação de Amparo à Pesquisa do Estado do Rio Grande do Sul 11/20722), the CNPq (475882/2012-1), and the PROBITEC-CAPES (004/2012).

\section{References}

[1] B. P. Chadwick and A.-M. Frischauf, "The CD39-like gene family: identification of three new human members (CD39L2, CD39L3, and CD39L4), their murine homologues, and a member of the gene family from Drosophila melanogaster," Genomics, vol. 50, no. 3, pp. 357-367, 1998.

[2] J. J. Mulero, G. Yeung, S. T. Nelken, and J. E. Ford, "CD39L4 is a secreted human apyrase, specific for the hydrolysis of nucleoside diphosphates," Journal of Biological Chemistry, vol. 274, no. 29, pp. 20064-20067, 1999.

[3] J. J. Mulero, G. Yeung, S. T. Nelken, J. M. Bright, D. W. McGowan, and J. E. Ford, "Biochemical characterization of CD39L4," Biochemistry, vol. 39, no. 42, pp. 12924-12928, 2000. 
[4] J. G. Páez, J. A. Recio, A. Rouzaut, and V. Notario, "Identity between the PCPH proto-oncogene and the CD39L4 (ENTPD5) ectonucleoside triphosphate diphosphohydrolase gene," International Journal of Oncology, vol. 19, no. 6, pp. 12491254, 2001.

[5] J. A. Recio, N. Zambrano, L. de La Pena et al., "cDNA isolation, expression, and chromosomal localization of the mouse pcph proto-oncogene," Molecular Carcinogenesis, vol. 26, no. 2, pp. 130-136, 1999.

[6] J. A. Velasco, R. Castro, M. A. Avila et al., "cph, a novel oncogene which cooperates with H-ras in the transformation of NIH3T3 fibroblasts," Oncogene, vol. 9, no. 7, pp. 2065-2069, 1994.

[7] J. A. Velasco, M. A. Avila, and V. Notario, "The product of the cph oncogene is a truncated, nucleotide-binding protein that enhances cellular survival to stress," Oncogene, vol. 18, no. 3, pp. 689-701, 1999.

[8] J. A. Recio, J. G. Páez, B. Maskeri, M. Loveland, J. A. Velasco, and V. Notario, "Both normal and transforming PCPH proteins have guanosine diphosphatase activity but only the oncoprotein cooperates with ras in activating extracellular signal-regulated kinase ERK1," Cancer Research, vol. 60, no. 6, pp. 1720-1728, 2000.

[9] C. M. MacCarthy and V. Notario, "The ENTPD5/mt-PCPH oncoprotein is a catalytically inactive member of the ectonucleoside triphosphate diphosphohydrolase family," International Journal of Oncology, vol. 43, no. 4, pp. 1244-1252, 2013.

[10] O. M. Tirado, S. Mateo-Lozano, S. Sanders, L. E. Dettin, and V. Notario, "The PCPH oncoprotein antagonizes the proapoptotic role of the mammalian target of rapamycin in the response of normal fibroblasts to ionizing radiation," Cancer Research, vol. 63, no. 19, pp. 6290-6298, 2003.

[11] R. Read, G. Hansen, J. Kramer, R. Finch, L. Li, and P. Vogel, "Ectonucleoside triphosphate diphosphohydrolase type 5 (Entpd5)-deficient mice develop progressive hepatopathy, hepatocellular tumors, and spermatogenic arrest," Veterinary Pathology, vol. 46, no. 3, pp. 491-504, 2009.

[12] A. Rouzaut, J. A. Recio, and V. Notario, "Expression of the protein product of the $\mathrm{PCPH}$ proto-oncogene in human tumor cell lines," Radiation Research, vol. 155, no. 1, pp. 181-187, 2001.

[13] M. Solanas, E. Escrich, A. Rouzaut, I. Costa, A. Martínez, and V. Notario, "Deregulated expression of the PCPH protooncogene in rat mammary tumors induced with 7, 12dimethylbenz[a] anthracene," Molecular Carcinogenesis, vol. 33, no. 4, pp. 219-227, 2002.

[14] A. Beckenkamp, D. B. Santana, A. N. Bruno et al., "Ectonucleotidase expression profile and activity in human cervical cancer cell lines," Biochemistry and Cell Biology, vol. 92, no. 2, pp. 95104, 2014

[15] S. Zadran, A. Amighi, E. Otiniano, K. Wong, and H. Zadran, "ENTPD5-mediated modulation of ATP results in altered metabolism and decreased survival in gliomablastoma multiforme," Tumour Biology, vol. 33, no. 6, pp. 2411-2421, 2012.

[16] J. Villar, M. I. Arenas, C. M. MacCarthy, M. J. Blánquez, O. M. Tirado, and V. Notario, "PCPH/ENTPD5 expression enhances the invasiveness of human prostate cancer cells by a protein kinase C $\delta$-dependent mechanism," Cancer Research, vol. 67, no. 22, pp. 10859-10868, 2007.

[17] J. Regadera, M. J. Blánquez, P. González-Peramato et al., "PCPH expression is an early event in the development of testicular germ cell tumors," International Journal of Oncology, vol. 28, no. 3, pp. 595-604, 2006.
[18] M. J. Blánquez, M. I. Arenas, I. Conde, O. M. Tirado, R. Paniagua, and V. Notario, "Deregulated expression of the PCPH proto-oncogene in human breast cancers," International Journal of Oncology, vol. 25, no. 4, pp. 821-830, 2004.

[19] M. J. Blánquez, J. Regadera, J. Mariño, R. E. Newman, and V. Notario, "Gradual deregulation and loss of PCPH expression in the progression of human laryngeal neoplasia," Molecular Carcinogenesis, vol. 35, no. 4, pp. 186-195, 2002.

[20] M. Mikula, T. Rubel, J. Karczmarski, K. Goryca, M. Dadlez, and J. Ostrowski, "Integrating proteomic and transcriptomic high-throughput surveys for search of new biomarkers of colon tumors," Functional and Integrative Genomics, vol. 11, no. 2, pp. 215-224, 2011.

[21] J. A. Recio, J. G. Páez, S. Sanders, T. Kawakami, and V. Notario, "Partial depletion of intracellular ATP mediates the stresssurvival function of the PCPH oncoprotein," Cancer Research, vol. 62, no. 9, pp. 2690-2694, 2002.

[22] J. Villar, H. S. Quadri, I. Song, Y. Tomita, O. M. Tirado, and V. Notario, "PCPH/ENTPD5 expression confers to prostate cancer cells resistance against cisplatin-induced apoptosis through protein kinase $\mathrm{C} \alpha$-mediated Bcl-2 stabilization," Cancer Research, vol. 69, no. 1, pp. 102-110, 2009.

[23] M. Fang, Z. Shen, S. Huang et al., "The ER UDPase ENTPD5 promotes protein N-glycosylation, the Warburg effect, and proliferation in the PTEN pathway," Cell, vol. 143, no. 5, pp. 711724, 2010.

[24] Z. Shen, S. Huang, M. Fang, and X. Wang, "ENTPD5, an endoplasmic reticulum udpase, alleviates ER stress induced by protein overloading in AKT-activated cancer cells," Cold Spring Harbor Symposia on Quantitative Biology, vol. 76, pp. 217-223, 2011.

[25] N. L. Curry, M. Mino-Kenudson, T. G. Oliver et al., "Pten-null tumors cohabiting the same lung display differential AKT activation and sensitivity to dietary restriction," Cancer Discovery, vol. 3, no. 8, pp. 908-921, 2013. 


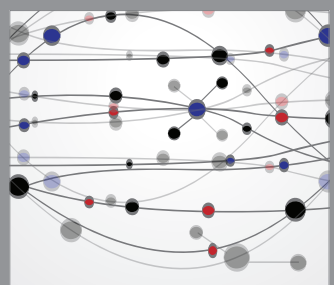

The Scientific World Journal
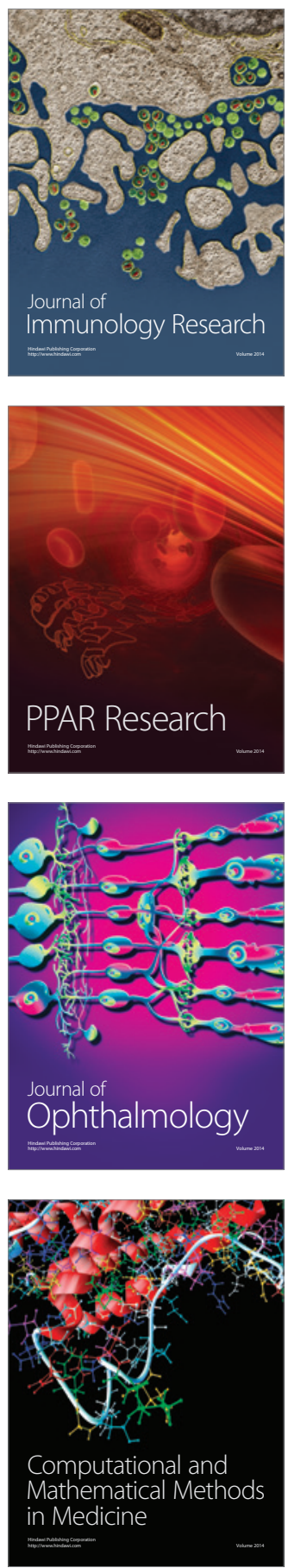

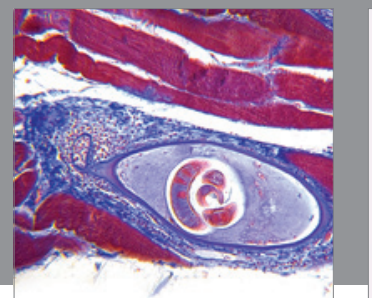

Gastroenterology

Research and Practice
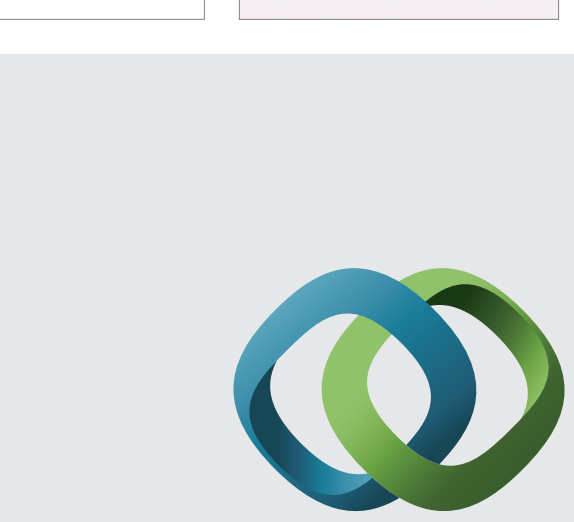

\section{Hindawi}

Submit your manuscripts at

http://www.hindawi.com
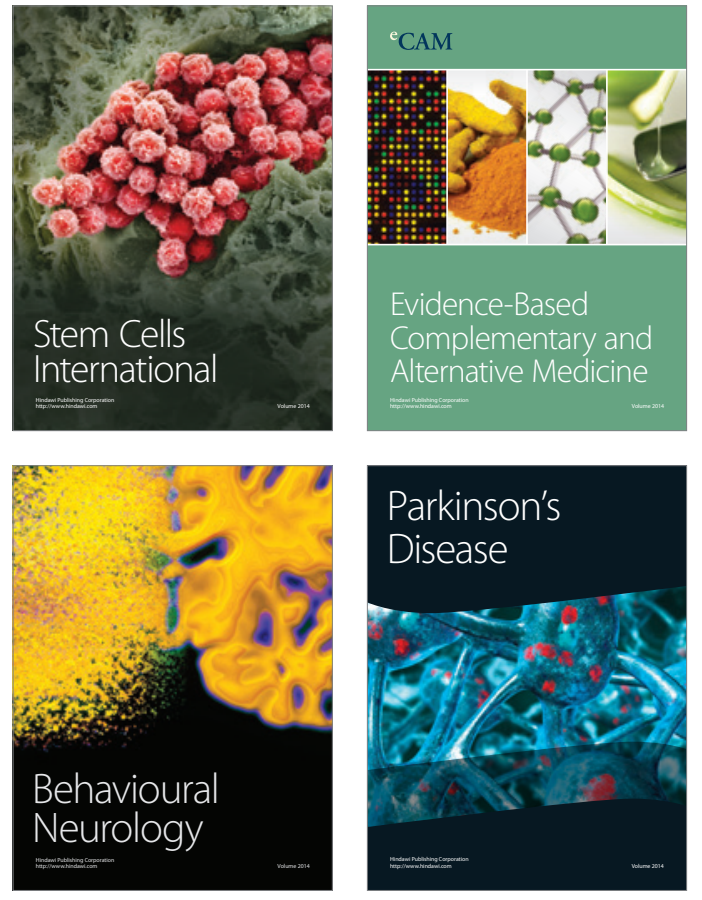
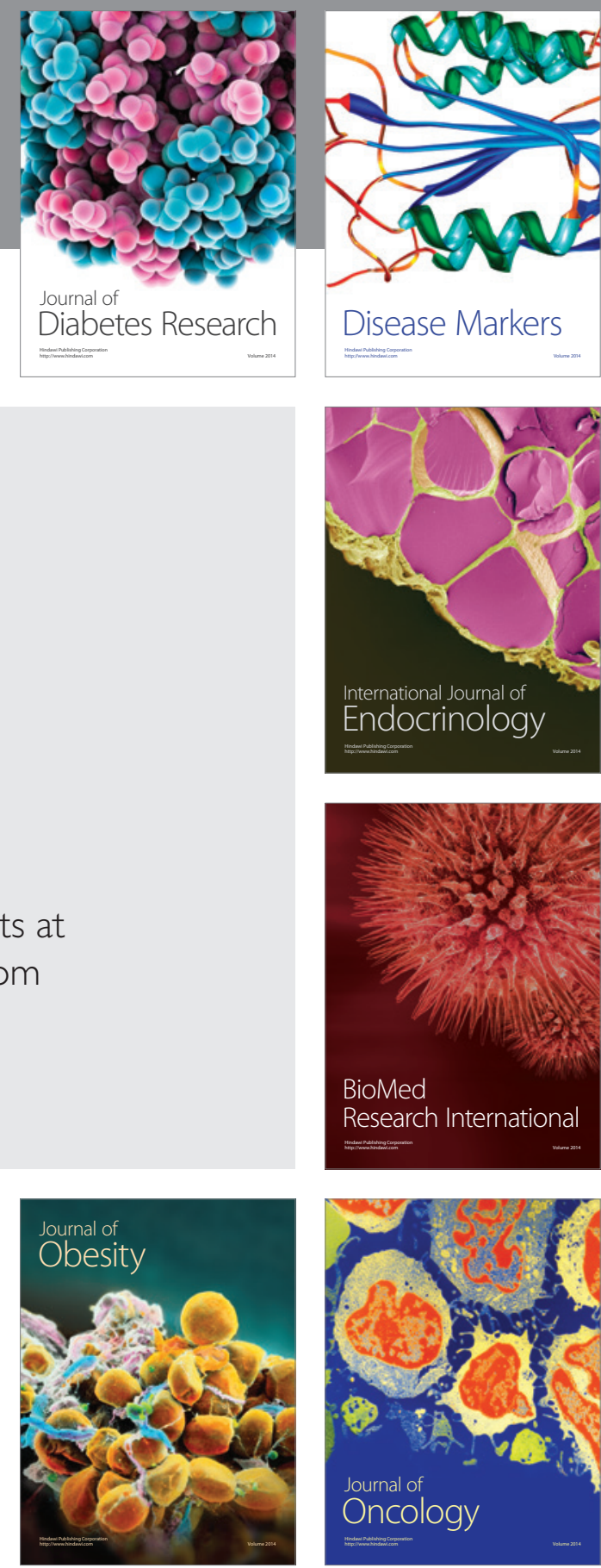

Disease Markers
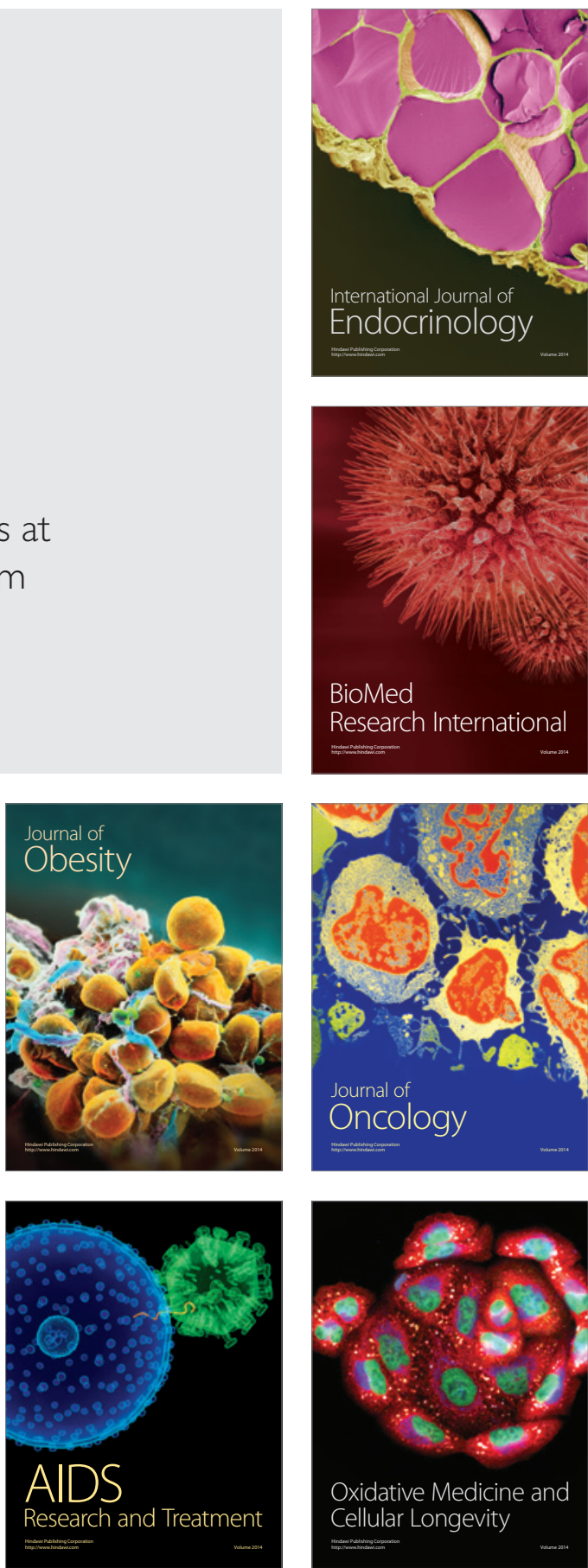